Slavica

bruxellensia

\section{Slavica bruxellensia}

Revue polyphonique de littérature, culture et histoire slaves

$1 \mid 2008$

Théâtre

\title{
Entretien avec Marian Pankowski
}

\section{Dorota Walczak et Jeremy Lambert}

\section{OpenEdition}

Journals

Édition électronique

URL : http://journals.openedition.org/slavica/236

DOI : $10.4000 /$ slavica.236

ISSN : 2034-6395

Éditeur

Université libre de Bruxelles - ULB

Édition imprimée

Pagination : $42-53$

ISSN : 2031-7654

\section{Référence électronique}

Dorota Walczak et Jeremy Lambert, «Entretien avec Marian Pankowski », Slavica bruxellensia [En ligne], 1 | 2008, mis en ligne le 15 octobre 2008, consulté le 20 avril 2019. URL : http:// journals.openedition.org/slavica/236 ; DOI : 10.4000/slavica.236

Ce document a été généré automatiquement le 20 avril 2019

\section{(c) (i) $\odot$}

Les contenus de Slavica bruxellensia sont mis à disposition selon les termes de la Licence Creative Commons Attribution - Pas d'Utilisation Commerciale - Pas de Modification 3.0 France. 


\title{
Entretien avec Marian Pankowski
}

\author{
Dorota Walczak et Jeremy Lambert
}

\section{Présentation}

1 Marian Pankowski est né le 9 novembre 1919 à Sanok, dans les Carpates polonaises. Fils d'un métallurgiste, il a passé son enfance et son adolescence dans sa ville natale, une petite bourgade habitée par des Polonais, des Juifs et des Ukrainiens. La guerre interrompit ses études de philologie à l'Université de Cracovie. Il prit part aux combats de l'armée polonaise. Arrêté en 1942 pour faits de résistance, il fut envoyé à Auschwitz. Transféré dans différents camps, la Libération le trouva à Bergen-Belsen, d'où il rejoindra Bruxelles. Il se fixa dans la capitale belge, où il poursuivit ses études. Il consacra sa thèse de doctorat au poète symboliste Bolesław Leśmian (1878-1937).

Devenu professeur, il a enseigné la littérature et la langue polonaises à l'ULB. Spécialiste de la littérature polonaise moderne, il s'est intéressé dans ses recherches à la " poésie brute » (couplets de noces, dictons, jurons, formules magiques).

Il a publié des recueils de poèmes, des récits, des romans et des pièces de théâtre. Celles-ci ont été mises en scène en France, en Belgique, en Suisse et surtout en Pologne. Depuis la Libération, il habite Bruxelles.

Une soirée d'auteur lui fut consacrée à l'ULB le 9 mai 2005.

\section{Entretien}

\section{Autodéfinition}

\begin{abstract}
Jeremy Lambert: Comment définiriez-vous votre écriture? Quels sont les éléments conscients et inconscients qui la caractérisent?

Il m'est possible de désigner les éléments conscients. Dans mes poèmes lyriques, je communiquais mes confidences, je disais mes émotions, grâce à une poétique classique où tout était lisible et transparent. La situation a changé lorsque j'ai quitté les strophes pour la prose, quand mes phrases commencèrent à charrier images, proverbes et mots
\end{abstract}


des faubourgs, mots qui n'étaient pas prévus, ni indispensables, mais qui m'envahissaient, avec ma complicité instinctive. Ma mémoire s'ouvrait et ces mots inattendus, ces mots risqués, parfois grossiers, venaient à la place d'un terme objectif et prévisible. J'en fus conscient assez rapidement. Et j'ai appelé cet envahissement jubilatoire « moja proza nie proza » (« ma prose qui n'en est pas»).

Dorota Walczak: Comment qualifieriez-vous vos inspirations? Stables ou instantanées, recueillies par surprise ou savoureusement recherchées?

Moi aussi, Madame, je me suis déjà posé cette question. Et il n'était pas facile d'y répondre, mais je vous dirais ce que je constate. J'y vois un dénominateur commun : ce serait mon indignation, ma révolte intérieure contre toutes les injustices sociales, morales ou sexuelles. Il m'est d'ailleurs un peu difficile d'en parler.

D. W. : Faites-vous partie de ces auteurs qui se promènent toujours avec un carnet de notes? Quelle est l'importance des détails dans votre écriture?

Non, je n'ai pas de carnet. Il me suffit d'un bout de papier pour noter un détail frappant. Il y a trois jours, dans mon restaurant favori, j'ai aperçu, trois tables plus loin, une jeune femme dont les yeux, la bouche et le nez étaient mal disposés dans son visage, ils étaient situés n'importe comment. Voici un détail que j'ai noté tout de suite sur ma serviette. Je le dévoile ici pour la première fois, mais je vais assurément l'utiliser un jour.

J. L. : Vos œuvres sont très différentes les unes des autres. Qu'est-ce qui constitue la matrice de l'écriture de Pankowski ? Cela a-t-il un lien avec la mention du lieu et de la date que l'on trouve souvent à la fin de vos textes?

C'est vrai, je veux que chaque nouveau livre soit différent du texte précédent. C'est vrai, c'est une obsession chez moi, et ce le fut dès le début. Quant à la date que je mets à côté de ma signature à la fin d'un texte, c'est peut-être une façon de m'inscrire dans le temps des hommes, de vouloir durer. C'est peut-être une coquetterie d'artiste...

D. W. : Je trouve qu'au niveau du style, une unité est bien repérable, un «style Pankowski » bien définissable : la richesse du vocabulaire, le côté baroque, «le haut » mélangé avec «le bas », donc le carnavalesque, la mémoire et l'histoire, l'actualité des faits divers... Vous utilisez la poétique très moderne du collage, la langue est traitée à la manière du XVIIe siècle : un coup de fouet et puis une caresse... à la langue.

Je suis heureux, Madame, d'entendre une évocation lapidaire, et si agréable, de mon style. Merci.

J. L. : Vous avez enseigné la littérature polonaise. La notion de «mémoire » est indissociable de celle d'enseignement. En tant qu'exilé, donnez-vous une teneur particulière à ce terme?

Je n'ai pas été un exilé. Je n'ai pas perdu ma patrie, je n'ai pas oublié ma langue polonaise. Le contenu de ma mémoire était polonais, mais ma méthode d'enseigner était belge! J'ai suivi l'exemple de mon patron, le professeur Claude Backvis ${ }^{1}$ : il analysait les œuvres des poètes romantiques polonais en séparant l'art et les qualités de la poétique du discours patriotico-religieux dominant dans la création de ces grands exilés. C'est ça qui m'a protégé. Ce fut un antidote, et grâce à lui, je n'ai pas acquis la mentalité des émigrés que je qualifierais de professionnels. Le fait que l'ULB était une sorte d'île de la réflexion, anti-sentimentale, m'a peut-être sauvé. Je pense que si j'avais habité une autre ville, j'aurais été pleurnichant, j'aurais chanté l'hymne national comme les autres et j'aurais finalement parlé de Varsovie comme les Juifs parlent de 
Jérusalem. Ce fut vraiment une grande chance de tomber sur la seule section de Slavistique qu'il y avait à l'époque en Belgique.

D. W. : Vous étiez pendant longtemps un écrivain «dérangeant», trop novateur, trop libre pour être publié en Pologne après la Deuxième Guerre mondiale. D'autre part vous étiez celui qui continuait, malgré les conseils, à voyager en Pologne communiste... Aujourd'hui la situation a changé. Vos livres sont de nouveau imprimés, les journalistes se succèdent pour vous interviewer... Comment ressentez-vous cette situation?

C'est un grand bonheur d'être accueilli en Pologne avec mes livres anciens et nouveaux. D'abord par un éditeur loyal, puis par des journalistes réellement intéressés par mon travail. Mais ce sont surtout les jeunes lecteurs qui me donnent le plus de satisfaction, surtout lors de mes rencontres à Cracovie et à Varsovie à l'occasion de la parution de Rudolf ${ }^{2}$, de PAtnicy $z$ Macierzyzny ${ }^{3}$ et de Ostatni zlot aniołów $w^{4}$. Je pourrais conclure : il y a des rêves qui se réalisent.

\section{Auschwitz}

J. L. : Vous avez connu les camps de concentration. Dans Z Auszwicu do Belsen ${ }^{5}$, vous écrivez «Mais là-bas... quand j'y étais, que j'y vivais... j'observais tout cela au nom de la LANGUE ${ }^{6}$. Vous définissez-vous ontologiquement comme un être parlant ?

Je n'entrerai pas dans la terminologie philosophique. Je pense que quand j'avais quinze ans, quand j'étais amoureux, j'ai pris la décision de devenir écrivain, de devenir poète. Et en quelque sorte, j'ai apporté cette religion à Auschwitz. Grâce à elle, je me suis considéré non pas comme un patriote malheureux, mais comme un poète qui a la chance de visiter un camp de concentration, de voir quelque chose d'extraordinaire, et... je crois que j'étais conscient du caractère exceptionnel de notre condition, de celle des dizaines de milliers de prisonniers qui étaient là. J'étais peut-être le seul qui se promenait en réfléchissant à notre situation, non pas en tant que patriote qui souffre, mais en tant qu'orgueilleux jeune poète qui se dit «moi je vais parler de tout ça ». Ma pensée allait assez loin : je voulais décrire Auschwitz en octaves. Et je le fis : en 1946, l'hebdomadaire "WIADOMOŚcI», basé à Londres, a publié mon poème intitulé « Auschwitz ».

Il faut aussi dire que je n'arrête pas de me chamailler, sur le plan linguistique, avec ceux qui utilisent improprement des termes importants. Quand je suis en Pologne, j'entends des phrases terribles: par exemple, pour qualifier les prisonnières d'Auschwitz, j'ai entendu l'expression «nasze oświęcimianki »! C'est comme si on parlait des membres d'un club sportif féminin! Il faut absolument faire des distinctions, il faut séparer les idées! La ville polonaise d'Oświęcim n'est pas coupable d'avoir accueilli un camp de concentration appelé Auschwitz. Ce sont les Allemands qui ont parachuté ce cancer de camps. Finalement, cela est associé à la Pologne dans son ensemble. Je crois qu'une certaine hostilité provient de cette imprécision.

D. W. : Z Auszwicu do Belsen a été nominé pour le prix Nike en 2001. Un livre si petit par ses dimensions et si grand dans son message et le poids émotionnel... Étiez-vous surpris par la réaction suscitée par ce livre dans les milieux des critiques, des Juifs, des Polonais, des Allemands... ?

Je suis heureux, et consolé, Madame, d'entendre votre présentation de mon petit livre. Je l'ai écrit cinquante ans après la libération des camps. Il m'a fallu cette marge pour oser présenter un texte libre, serein, et libre d'une quelconque censure. Il fallait aussi 
que pendant ce temps, l'étudiant rêveur devienne l'homme d'Auschwitz, que son écriture soit apte à aborder un sujet aussi dantesque.

J. L. : Comment s'impose-t-on dans la littérature des camps après la triade Krall - Herling Borowski?

Les écrivains polonais qui ont connu Auschwitz ont tous traité ce sujet comme un témoignage. Ils étaient là, ils ont vu, ils ont constaté et ils séparent d'une manière, que je qualifierais de manichéenne, les victimes et les bourreaux. Pour moi, ce qui compte c'est la qualité littéraire d'un texte, d'une œuvre. Personnellement, j'apprécie beaucoup les récits de Tadeusz Borowski. Je trouve qu'il s'y exprime avec sobriété tout en s'écartant de toute flatterie patriotique. Et il est dur. En analysant ses récits avec mes étudiants à l'Université, nous avons constaté qu'il avait « densifié » les événements. Par exemple, dans Dzień na Harmenzach ${ }^{7}$, il a groupé plusieurs instants vécus à des moments différents, cette journée est donc infernale, tout s'y passe. Evidemment, on peut trouver cette densité excellente, on peut la qualifier d'irréaliste, mais moi j'ai apprécié.

D. W. : Je crois encore une fois qu'avec Z Auszwicu do Belsen vous avez franchi un tabou. Vous avez abordé le sujet comme personne ne l'avait fait avant vous. Impossible de déposer ce livre, de faire une pause, il le faut lire d'une traite... II y a la tragédie, l'impensable si triste et poignant dans sa vérité, mais il y a aussi de l'humour... inconcevable?

Rien n'a faire, il faut à nouveau que je blasphème. Mon aveu peut surprendre et même choquer : l'exil a été pour moi une chance. Transféré d'Auschwitz à Gross-Rosen, de Gross-Rosen à Northausen, de Northausen à Bergen-Belsen, et libéré après trois années du pyjama rayé, j'ai continué le voyage vers Bruxelles avec ma tuberculose et mes 55 kilos pour 1m84. Guéri, nourri, j'ai reçu la carte d'étudiant de l'ULB. Étudier en Occident... «Zachód»! Ce mot, pour un Polonais cultivé, appelle des associations d'idées favorables. C'est un peu la cour du mythique royaume d'Europe, avec son catholicisme millénaire et son latin, qui, dès le $\mathrm{X}^{\mathrm{e}}$ siècle, a servi de modèle à la culture et à la langue polonaises, qui, au XVI ${ }^{\text {e }}$ siècle, a favorisé l'essor des lettres et de la pensée polonaises, cet essor que les Polonais appellent l' «Âge d'or ». Défendre ma thèse sur Leśmian dans une Université occidentale, y enseigner ensuite la langue et la littérature de mon pays, est-ce une suite d'heureux hasards? Qui pourrait nier que ce fut une véritable chance?

\section{Maîtrise de la langue} J. L. : Cette maîtrise de la langue qui vous caractérise est-elle une conséquence directe de
votre qualité d'exilé ? Est-elle la preuve active de votre polonité en risque de deuil?

La réponse est oui et non. Ou plutôt c'est un oui auquel manque quelques détails. Il est vrai que j'ai connu des moments de crainte, notamment de perdre la maitrise de mon polonais. J'ai toujours été orgueilleusement fier de ma connaissance du polonais, déjà lorsque j'étudiais à l'Université de Cracovie. Pour sauver mon polonais, pour le soigner, j'ai utilisé plusieurs techniques. Je faisais notamment de nombreux voyages oniriques avant de m'endormir. Je me voyais déambuler dans ulica (rue) Niecała, par exemple, je visionnais la petite maison de Gruba (grosse) Anielka, la prostituée municipale, je me faisais un itinéraire, je passais par telle ou telle rue, je m'entretenais avec les voisins, j'épuisais ainsi toutes les maisons en m'endormant. Le polonais qui me revenait était vivant, parlant et émouvant en même temps. Je faisais ces voyages oniriques uniquement dans ma ville natale, nulle part ailleurs, parce que je connaissais pour ainsi dire chaque pierre. 
D. W. : Dans vos écritures apparaît aussi de façon perceptible le soin de la parole, de la littérature lue à haute voix, l'art sublime de la conversation... Ceux qui ont la chance de vous entendre parler le confirmeront. Cultivez-vous en pleine conscience cet antique art de la "gawęda » $^{8}$ ?

Tout à fait. D'ailleurs, aux techniques que je viens de citer, il faut ajouter ceci : de temps en temps, je rapportais chez moi de l'ULB un volume du Wielki Słownik JĘzyka Polskiego (Grand dictionnaire de la langue polonaise), on disait alors Słownik Warszawski (Dictionnaire varsovien), et je le lisais à haute voix dans mon bureau lorsque ma femme et ma fille dormaient. Je lisais uniquement pour m'entendre. Et puis, il y avait les huit heures de mon lectorat. C'était un boulot mal payé, mais grâce à lui, je conservais mon polonais puisque je devais parler à voix haute huit heures par semaine. De plus, chaque année, je passais une dizaine de jours en Pologne et je notais les mots qui me frappaient, qui étaient nouveaux pour moi. Je vous citerai un exemple marrant. J'étais en Pologne, ce n'était déjà plus un pays communiste, elle ne connaissait plus cet ordre particulier dans lequel tout semblait marcher. Bien sûr, on était surveillé, mais tout ne semblait pas anarchique, contrairement à l'époque qui suivit la Table Ronde. Un taxi, conduit par une personne assez âgée, était venu me chercher à l'aéroport. À la vue des cheveux gris du taximan, je me suis dit qu'il connaissait la vie, et j'ai entamé une conversation avec lui. À un moment, je lui demande si son métier connaît la crise. Il me répondit: « Mais oui, Monsieur ! Avant on avait des horaires, des véhicules, des points, etc. Alors que maintenant, kurwa wszystko puścili na żywioł!» (trad. : Putain, ils ont tout laissé foutre le camp. Litt. : ils ont tout laissé partir vau-l'eau). Le mot «Żywioł», "l'élément ", évoquait en moi quelque chose de mythique, d'astronomique, l'immense phénomène de la nature ! Et pour lui, cela évoquait le bazar, le bordel, tout ce qui fout le camp. C'étaient des termes comme celui-ci que je ramenais de Pologne. Aujourd'hui, ce qui caractérise mes livres, ce n'est pas ce que disent les journalistes, «jĘzyk polski », la «langue polonaise", accompagné d'un adjectif élogieux, mais plutôt "mowa polska », le "parler polonais ». C'est dans ce "parler» mâtiné d'ukrainien et d'autrichien que je puise. Ma femme, qui était originaire de Lvov, était juive et avait dû se cacher à Varsovie, c'est elle qui m'a appris beaucoup de mots varsoviens. Quand je désignais la ciboulette, j'utilisais un mot de Sanok, je disais «trymbulka ». Elle m'a appris qu'à Varsovie, on disait «szczypiorek». Aujourd'hui que je vis seul, oui, je pratique encore l'art de la gawĘda pour garder le vocabulaire et la prononciation.

J. L. : Ce qui me marque personnellement dans votre écriture, c'est la qualité de vos ellipses, entre silence, sous-entendus et non-dits...

J'apprécie votre analyse de mon écriture, votre lecture consciente de mes sousentendus et de mes non-dits. En écrivant, je ne forme pas sciemment ma phrase, elle coule, elle vient, et c'est ainsi qu'elle ramasse dans ma tête tout ce qui passe en associations, en fantasmes, etc.

D. W. : Vous pouvez aussi être perspicace et pointu dans vos propos. Evoquons Rudolf par exemple. Vous êtes d'ailleurs devenu, après la réédition de ce roman et les interviews (visite chez « Madame »), l'auteur coté du milieu gay en Pologne ? Et... ?

C'est un concours de circonstances bien étrange, la réédition de mon Rudolf par Piotr Marecki parut à Cracovie en 2005, au moment où les homosexuels polonais sortaient de la clandestinité. Ce fut un hasard de rencontrer un éditeur qui a voulu, qui a osé, rééditer mon livre. D'ailleurs, deux ans plus tard, une quatrième édition a vu le jour. C'est unique, n'est-ce pas, dans la Pologne que nous connaissons... 


\section{Traduction}

J. L.: Votre œuvre a été traduite presque entièrement. Quel est votre rapport aux traducteurs? Habitant en francophonie, pensez-vous à la traduction lorsque vous écrivez ?

Jamais. Je me souviens d'une discussion avec Jerzy Giedroyć, le rédacteur de la revue parisienne «KULTURA", il me disait à peu près ceci: "vous savez, j'ai beaucoup d'admiration pour Gombrowicz, mais ce qui me gêne, c'est qu'il veut plaire aux Occidentaux en écrivant des livres dont le climat n'est pas polonais. » Moi, je n'ai jamais été contraint de me comporter comme les écrivains qui guettent les occasions, j'étais libre, personne ne m'attendait, personne ne savait ce que j'écrivais. Heureusement j'avais accès, en Belgique, à des traducteurs qui sont tous d'anciens slavisants de notre Université. Et quand ils traduisent mes livres, ils ont l'amitié de me soumettre leur travail. Il arrive que je leur signale un régionalisme ou un néologisme de ma fabrication. Dans ces cas-là, je les aide, mais en général, ils connaissent bien mon polonais.

D. W. : À côté de votre production littéraire propre, vous avez traduit de nombreux poèmes polonais en français. Le polonais exprime bien la complexité du traduire par nécessité, on dit «nie jestem, ale bywam», «je ne le suis pas mais il m'arrive de l'être ». Comment vous sentez-vous dans le rôle de traducteur?

Mes traductions ont été stimulées par des moments très différents dans ma vie. J'ai publié une anthologie de la poésie polonaise pour laquelle j'ai traduit moi-même les poèmes. Lorsque je travaillais à l'ULB, Claude Backvis parlait de la poésie polonaise avec énormément de chaleur et d'émotion, les adjectifs flatteurs s'accumulaient dans ses explications. Mais quand il fallait proposer une strophe, la traduction n'était pas à la hauteur des poèmes cités. Les étudiants acceptaient cela comme une illustration un peu maladroite de la poésie polonaise. Je me suis alors dit qu'il fallait que les étudiants puissent lire cette poésie chez eux. J'ai donc fait un choix de poèmes parmi ceux qui me plaisaient personnellement, du XV $\mathrm{XV}^{\mathrm{e}}$ siècle jusqu'à Miłosz, et je les ai traduits. J'ai ensuite élargi cette sélection. En Pologne populaire, il y avait une anthologie très intéressante de Jan Kott et de Adam Ważyk intitulée Wiersze, które lubimy ${ }^{9}$ (trad. : Les vers que nous aimons), j'en ai tiré des poèmes engagés, que je n'utilisais pas dans mes recherches mais que je jugeais intéressants. Cette anthologie a été publiée à compte d'auteur chez un éditeur nommé André Derache, installé à Aalter, en Belgique. Mon premier stimulant dans cette publication était que les étudiants de slavistique puissent lire la poésie polonaise, comme cela existait depuis longtemps dans le domaine russe. C'était d'ailleurs la première anthologie de poésie polonaise traduite en français.

Ensuite, j'ai traduit des poèmes allemands. J'avais rencontré un couple d'Allemands pendant des vacances familiales en Italie, le monsieur, très catholique, était poète. Nous avons bavardé et il m'a proposé de traduire son livre, qui racontait l'histoire de soldats allemands qui ont survécu durant cinq années dans un bunker en Poméranie. Les enfants qui jouaient au-dessus d'eux entendirent des coups qui provenaient du sol, on a parlé de fantômes, d'esprits, etc. Finalement les sapeurs polonais ont ouvert ces casemates, et cinq hommes en sont sortis. Quatre sont morts directement au choc de la lumière et le dernier fut sauvé... pour exister plus tard dans mon roman Fara na Pomorzu ${ }^{10}$. Ces hommes avaient véritablement vécu le martyre, ils n'ont été sauvés que parce qu'il étaient magasiniers de nourriture... Un officier qui vivait parmi eux s'était suicidé, ils l'avaient enterré dans le riz. 
Enfin, j'ai traduit des poèmes de Leśmian écrits en russe, des poèmes de ses débuts. Il faut dire qu'il baignait dans la culture russe. Dans ses premiers poèmes polonais, il fit d'ailleurs des erreurs de syntaxe, il écrivait à la russe. J'ai publié la traduction de ses deux cycles de poèmes russes en Pologne.

J. L. : Relisez-vous vos œuvres traduites?

Je pense que cela fait partie de la relation entre l'auteur et le traducteur. Je lis la version française en tant qu'écrivain qui connaît l'intention de l'auteur.

J. L. : Vous avez été traduit par Alain van Crugten, qui a été un de vos collègues à l'Université. Comment concilie-t-on l'idée de collègue (considéré sur un pied d'égalité) et de traducteur (esclave du texte qu'il traduit)?

Cette image est un peu trop forte. La réalité de la relation entre l'auteur et le traducteur que vous citez philosophiquement était toute différente. Le traducteur de mon Rudolf venait avec sa machine à écrire, je m'asseyais en face de lui. Il avait le texte polonais devant lui, le lisait à haute voix et en donnait la traduction, puis, s'il n'y avait de ma part aucune remarque, il l'écrivait directement. Ça venait très vite. Parfois il s'arrêtait et cherchait un terme adéquat parmi une série de synonymes qu'il trouvait dans un gros dictionnaire, un dictionnaire excellent qui proposait une litanie de nuances. $\mathrm{Au}$ fond, je l'assistais en silence, j'intervenais rarement, uniquement pour signaler une expression idiomatique. Nous étions collègues en poésie, nous le sommes toujours... Je crois que j'ai détruit votre image diabolisée ! (rire)

\section{Figures littéraires}

J. L. : Parmi vos figures littéraires principales, on retrouve votre mère. Lorsque vous la faites parler, vous utilisez le même style, dans Rudolf et dans Nastka, śmiej się ${ }^{11}$, notamment. Le référent de la figure littéraire est-il si fort que vous ne puissiez pas le modifier à l'envi ?

Ma mère est la seule figure, parmi celles que j'utilise, que je n'adapte pas. Vous avez bien remarqué l'identité du style dans Rudolf et dans Nastka, śmiej się. C'est son style, je l'impose comme littéraire. Elle vient dans mes récits directement de sa cuisine de Sanok, portant son tablier.

D. W. : C'est votre mère qui vous a donné la première, me semble t-il, le goût de la langue : puissante quand il le faut, sublime, charnelle, ou tout à la fois.

Nous abordons ici le sujet, souvent altéré, de la démarche initiatique de la mère. Mais je la trouve convaincante dans ce cas-ci. Imaginez-vous la mère qui s'approche de l'enfant qui s'éveille et lui demande de fermer les yeux. Elle dépose alors un lapereau qui vient de naître dans les mains de l'enfant et lui permet de regarder. Il trouve un lapereau, c'est une surprise pour lui. Un autre jour, elle dépose une Reine-Claude qui vient de tomber de l'arbre, encore chaude des rayons de soleil. Un autre jour encore, c'est un petit morceau des premières glaces, celles qui annoncent l'hiver, qu'elle lui dépose entre les doigts. L'enfant pousse des cris, bien sûr, mais comprend que c'est comme ça que commence l'hiver.

Je ne ferai toutefois pas une étude de la langue utilisée. Dans mes textes, dans mes livres, partout où ma mère apparait, j'utilise des expressions typiques, provenant des faubourgs ouvriers. Pour convaincre gentiment les journalistes qui viennent m'interviewer, je leur raconte cette histoire, en français, bien que cela fausse un peu l'effet. Nous sommes en avril, ma mère rentre avec des achats. Il y a au sol un tapis blanc de gel, et ma mère dit « Mon p'tit, ce salaud de gel est arrivé et les petits culs des 
violettes ont froid. » Ou celle-ci : À Sanok, lorsque les Allemands ont quitté la région, un bourgmestre a été élu, c'était un communiste fidèle. Mais il aimait la boisson, c'était un ivrogne. Ma mère m'écrivit alors une lettre dans laquelle elle disait: «Tu sais, c'est comme ça maintenant, les violettes sont gelées et les merdes fleurissent. » Il n'y avait pas de limites... C'étaient des lettres comme cela que je recevais chaque semaine. Je les ai toutes gardées, et je réfléchis à qui je pourrais les confier en Pologne, afin qu'elles soient utilisées dans le cadre d'une étude philologique. Uniquement dans ce cadre-là.

J. L. : Vous êtes souvent le "professeur » dans les mots de votre mère... que vous-même placez dans sa bouche. Faut-il y voir la cause dans la force de ce référent?

La question est fine et pas facile du tout parce qu'elle s'étend sur plusieurs domaines. Il faudrait peut-être un psychologue... mais je ne peux pas être appelé à écrire des livres et à en donner un commentaire psychologique, c'est beaucoup trop demander d'un auteur. C'est peut-être un trait caractéristique des gens pauvres, ils sont fiers de citer une situation avantageuse ou un titre de l'un des leurs, croyant que cela valorise leur démarche, que cela anoblit la famille. Mais je pourrais voir la naissance de mon texte autrement, considérer celui-ci comme de la prose composée d'éléments hétéroclites dans laquelle je situe un être en chair et en os. Je fais parler ma mère sans lui imposer mon vocabulaire, sans censurer les grossièretés de son langage des faubourgs ouvriers.

J. L. : Qu'est-ce qui se cache sous la figure littéraire du « professeur»? Une revanche sur la vie, sur la Pologne populaire, sur l'exil, sur la langue?

Il m'est difficile de décoder le pourquoi de l'emploi d'un mot, de sa présence dans mon discours. D'ailleurs je dois sans cesse me battre intérieurement pour ne pas utiliser mes connaissances intellectuelles, universitaires, ou mes lectures dans le « MONDE » parisien. Je me dis que j'ai mon univers, que je dois le vomir tel quel, sans ajouter de petites fleurettes parisiennes ou universitaires. Ce n'était pas facile, mais je crois que mon aspect sauvage de provincial des Carpates m'a toujours suffisamment protégé. J'aime adapter le contenu sémantique de ce mot, toujours le même, à des situations différentes. Je crois qu'on pourrait presque écrire un essai psychanalytique. Il y a assurément un rapport avec ma mère, pour elle, c'était une joie sans bornes d'avoir un fils professeur. Je crois avoir mérité ce titre, j’ai écrit une thèse de doctorat, finalement. Mon travail à l'Université m'a permis de découvrir le baroque polonais, que je ne connaissais pas du tout. J'en suis tombé amoureux, vous le constatez d'ailleurs vousmême.

\section{Théâtre}

J. L. : Votre écriture dramatique ne permet pas à l'acteur de s'approprier la totalité des éléments littéraires, je pense à des didascalies telles que « du champagne comme de l'urine tiédie $»^{12}$ ou «ils s'enfoncent jusqu'à la taille, jusqu'à la poitrine, puis ils grandissent sur notre vie terrestre ${ }^{13}$. J'ai l'impression que ce sont plutôt les termes, les concepts langagiers développés que les acteurs doivent s'approprier...

J'ai beaucoup apprécié votre analyse, elle est fine et très juste. Je pense que si je commets mes didascalies, c'est qu'elles sont présentes dans ma tête et sont appelées à la surface à un certain moment. Ma présence intervient soi-disant mine de rien, mais un lecteur comme vous la remarque tout de suite. Et vous n'êtes pas seul, Krystyna RutaRutkowska ${ }^{14}$ l'a remarquée également. Elle développe cette idée dans son travail de doctorat. Elle y a inséré un petit chapitre dédié aux didascalies, elle les évoque, les analyse et leur attribue un rôle actif, une présence comparable à celle des personnages. 
D. W. : Beaucoup de vos romans sont tellement théâtraux! Wyspiański pourrait être jaloux... Je pense à Bal wdów i wdowców ${ }^{15}$ (Le bal des veufs et des veuves), on trouve une intrigue shakespearienne, avec des poursuites et de la tromperie, et de la danse.Y aurait-il un clin d'œil au bal des débutantes?

Merci de citer le nom de Wyspiański. Je ne peux pas développer cela, on pourrait croire que nous sommes deux copains, Wyspiański et Pankowski. En ce qui concerne Bal wdówi wdowców, le thème en est imaginaire. Je me souviens qu'une amie journaliste voulait trouver la société organisatrice de ce bal, elle a cherché partout, dans l'annuaire, sur Internet, elle a même téléphoné au casino d'Ostende. Elle n'a rien trouvé... Vous soulignez le caractère théâtral de mes romans. Hé bien, mon éditeur français m'a refusé un roman, Balustrada ${ }^{16}$, qui a été traduit en français, mais n'a jamais été publié, parce qu'il jugeait ce roman trop théâtral. Peut-être avait-il raison (rire).

\section{En conclusion}

D. W. : Auriez-vous la gentillesse de dévoiler pour nos lecteurs un tout petit peu les secrets de vos nouveaux projets?

En octobre paraîtra à Varsovie mon nouveau texte, Była Żydówka, nie ma Żydówki (Il y avait une Juive, elle n'est plus là). Le texte a été publié l'année passée en août dans « TWóRCzość ». C'est un texte important, et j'ajouterais, sur un ton tristounet, comme tout ce qui paraît en Pologne au sujet des Juifs. Après la lecture de ce texte, un homme m'a écrit une lettre superbe. C'était un ancien président du consistoire juif de Belgique. Une lettre superbe, bouleversante. J'ai été élevé depuis ma tendre enfance parmi les Juifs et les Ukrainiens, je ressens une affection fraternelle pour eux. Ce nouveau texte est nuancé, c'est un texte où un Polonais qui vit en Belgique parle de la Pologne, c'est-àdire de nulle part, comme le disait Alfred Jarry, nulle part... mais là où il y a des Juifs et des Polonais. Je parle d'eux, j'évoque la judéité éternelle. Mon texte commence par cette idée qu'il y a deux nations, paradoxalement cosmopolites, qui portent fièrement le nom de leurs ancêtres : les Juifs et les aristocrates.

Dans mon tout nouveau texte, que je viens de donner à une journaliste pour qu'elle le retape sur son ordinateur, je me suis permis d'aller carrément vers un baroquisme que je qualifierais de personnel. Dans ce long roman, j'exagère, je suggère des situations équivoques, un peu louches, mais avec humour...

D. W. \& J. L. : Au nom de « SLAVICA BRUXELLENSIA », nous vous remercions pour cette cordiale interview qui nous a permis de voyager dans votre œuvre et dans le temps, ce temps que vous apprivoisez si bien.

\section{NOTES}

1. - Claude Backvis (1910-1998), éminent slavisant, devient assistant chargé des langues russe en polonaise à l'ULB en 1933. Durant la Deuxième Guerre mondiale, il assure des cours clandestins de 1940 à 1944. En 1945, il reprend son poste à l'ULB et qu'il garde jusqu'à sa retraite en 1977. Homme aux talents multiples, il fut élu à l'Académie des Sciences de Pologne. 
2. - Paru à Varsovie aux éditions Czytelnik en 1984. Édition française : Rudolf, éd. L'Âge d'Homme, Lausanne, 1982 (traduit par Alain van Crugten).

3. - Paru à Lublin aux éditions Wydawnictwo Lubelskie en 1987. Édition française : Les Pèlerins d'Utérie, éd. Actes Sud, Paris, 1986 (traduit par Elisabeth Destrée-Van Wilder).

4. - Paru à Varsovie aux éditions Wydawnictwo Krytyka Polityczna en 2007.

5. - Paru à Varsovie aux éditions Czytelnik en 2000. Édition française : D’Auschwitz à Bergen-Belsen. Aventures, éd. L'Âge d'Homme, Lausanne, 2000 (traduit par Yolande Lamy).

6. - D’Auschwitz à Bergen-Belsen, Op. cit., p. 31.

7. - Traduite du polonais par Laurence Dyèvre et Eric Veaux sous le titre Une journée à Harmenze, cette nouvelle a paru en français dans le recueil Le Monde de pierre (éd. Christian Bourgois, Paris 2002).

8. - GawĘda : récit oral prononcé lors des banquets qui suivaient la chasse dans la culture de la petite noblesse polonaise (szlachta). Elle devint un genre littéraire au XIX ${ }^{\mathrm{e}}$ siècle.

9. - Cette anthologie parut en 1951 à Cracovie aux éditions Czytelnik.

10. - Paru à Cracovie aux éditions Wydawnictwo Literackie en 1997. Édition française: Un monastère en Poméranie, Actes Sud, 1997 (traduit en français par Elisabeth Van Wilder).

11. - Nouvelle parue dans « LAMPA» (Varsovie) en décembre 2007.

12. - Capucine à Bécaussines, pièce traduite en français par Alain van Crugten et parue dans Marian Pankowski, Théâtre complet, t. 2, éd. L'Âge d'Homme, Lausanne, 1978, pp. 51-89. Édition polonaise : Pięć dramatów, Wydawnictwo Lubelskie, Lublin, 2001.

13. - Les Mâchoires d'or, pièce traduite en français par Alain van Crugten et parue dans Marian Pankowski, Théâtre complet, Op. cit., pp. 7-50. Edition polonaise : Pięć dramatów, Ibid.

14. - Krystyna Ruta-Rutkowska, Dramaturgia Mariana Pankowskiego: problemy poetyki dramatu współczesnego, (La dramaturgie de Marian Pankowski: problèmes de la poétique du drame contemportain, DiG, Varsovie, 2001, 219 p.

15. - Paru à Varsovie en 2006 aux éditions Korporacja Ha!art.

16. - Paru à Londres aux éditions Oficyna Poetów i Malarzy en 1996. Édition française : La balustrade, L'Âge d'Homme, 2004 (traduit en français par Elisabeth Destrée-Van Wilder).

\section{INDEX}

Index chronologique : XXe siècle

Index géographique : Belgique, Pologne

Mots-clés : émigration polonaise, littérature polonaise

\section{AUTEURS}

\section{DOROTA WALCZAK}

Responsable de la Chaire de Polonais, section de Langues et Littératures modernes, option Slaves, de l'Université Libre de Bruxelles (Belgique) ; rédactrice en chef de « Slavica Bruxellensia » 


\section{JEREMY LAMBERT}

Docteur en Langues et Littératures Slaves (Université Charles-de-Gaulle Lille3) ; membre du Centre d'Études en Civilisations, Littératures et Lettres Étrangères (CECILLE) de l'Université Charles-de-Gaulle Lille3; membre du comité de lecture et rédacteur technique de «Slavica Bruxellensia » 